\title{
20 years of the Cochrane Collaboration
}

\author{
Derek Richards \\ Editor
}

This year the Cochrane Collaboration is celebrating its $20^{\text {th }}$ anniversary. As part of this anniversary a series of 24 short videos have been produced and they are being shown one every two weeks during the year on the Cochrane anniversary site (http:// anniversary.cochrane.org).

The time line of the Cochrane Collaboration can be found on their website (http://www.cochrane.org/about-us/ history). This shows that the concept of the Collaboration was first presented at a conference ('Doing more good than harm') organised by Kenneth Warren and Frederic Mosteller at the New York Academy of Sciences, with the Collaboration being formally launched at the first Cochrane Colloquium in Oxford in November 1993. This year the $21^{\text {st }}$ colloquium is being held in Quebec in September. Prior to 1993 the time line highlights a number of events that pre-date the formal establishment of the Collaboration, the first being the publication of Archie Cochrane's book, Effectiveness and Efficiency: random reflections on health services. ${ }^{1}$ The book is currently out of print but a new edition is anticipated during the year. Further background on Archie Cochrane after whom the collaboration is named can be found on the Cochrane website together with a wealth of information about the Collaboration's activities.
Currently there are over 5000 systematic reviews on the Cochrane library, with a further 2000 protocols for reviews in progress. The Cochrane Oral Health Group is one of more than 50 review groups working to produce reviews for the library. The oral health group has over 600 authors for 40 different counties and currently over 140 oral health reviews are listed. While the systematic reviews produced by Cochrane are a key component of the library it also contains a number of other databases that add to this marvellous resource, a central register of controlled trials (CENTRAL), the Database of Reviews of Effectiveness (DARE) produced by the University of York, together with databases of methodological studies, technology assessments and economic evaluations.

As usual we have a number of commentaries in this month's journal from Cochrane reviews. In a break from the norm we have published two commentaries on the review by de Souza et al. ${ }^{2}$ on the management of temporomandibular joint osteoarthritis, to illustrate the slightly differing perspectives of different commentators.

1. Cochrane AL. Effectiveness and Efficiency. Random Reflections on Health Services. London: Nuffield Provincial Hospitals Trust, 1972. (Reprinted in 1989 in association with the BM], Reprinted in 1999 for Nuffield Trust by the Royal Society of Medicine Press, London (ISBN 1-85315-394-X).

2. de Souza RF, Lovato da Silva $\mathrm{CH}$, Nasser M, Fedorowicz Z, Al-Muharraqi MA. Interventions for the management of temporomandibular joint osteoarthritis. Cochrane Database Syst Rev 2012; Issue 4. Art. No. CD007261. DOI: 10.1002/14651858. CD007261.pub2

\section{Corrections and clarifications}

Spivakovsky S. Will adding acetaminophen (paracetamol) to ibuprofen be more effective in relieving postoperative pain on symptomatic necrotic teeth? Evid Based Dent. 2012; 13(4): 105.

Thanks to Julie Williams from Taunton who pointed out that we were less that clear about the intervention in our abstract of the above article. We stated

"The patients received a bottle containing either 80 capsules of $150 \mathrm{mg}$ ibuprofen or 80 capsules of $150 \mathrm{mg}$ ibuprofen/250 $\mathrm{mg}$ acetaminophen. The patients were then instructed to take 4 capsules every 6 hours as needed for pain."

Our summary of the intervention has oversimplified and we should have stated

....at the time of treatment were randomised to receive a bottle of to either 80 capsules of $150 \mathrm{mg}$ ibuprofen or 80 capsules of $150 \mathrm{mg}$ ibuprofen/250mg acetaminophen. Patients were to take four capsules every six hours.

Evidence-Based Dentistry (2013) 14, 2. doi:10.1038/sj.ebd.6400907. 